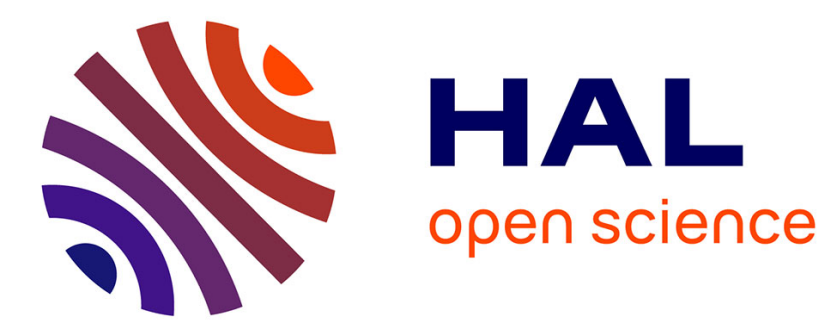

\title{
Flow of low pressure gas through dual-porosity media
}

J. Chastanet, Pascale Royer, J.L. Auriault

\section{To cite this version:}

J. Chastanet, Pascale Royer, J.L. Auriault. Flow of low pressure gas through dual-porosity media. Transport in Porous Media, 2007, 66, pp.457-479. 10.1007/s11242-006-0023-y . hal-00582929

\section{HAL Id: hal-00582929 \\ https://hal.science/hal-00582929}

Submitted on 29 Aug 2018

HAL is a multi-disciplinary open access archive for the deposit and dissemination of scientific research documents, whether they are published or not. The documents may come from teaching and research institutions in France or abroad, or from public or private research centers.
L'archive ouverte pluridisciplinaire HAL, est destinée au dépôt et à la diffusion de documents scientifiques de niveau recherche, publiés ou non, émanant des établissements d'enseignement et de recherche français ou étrangers, des laboratoires publics ou privés. 


\title{
Flow of low pressure gas through dual-porosity media
}

\author{
J. Chastanet - P. Royer - J.-L. Auriault
}

\begin{abstract}
Using the theory of homogenization we derive macroscopic models for describing flow of gas at low pressure in dual-porosity media. The case of a fractured porous medium is under consideration for the study, and the existence of a representative elementary volume that consists of open connected fractures surrounded by porous matrix blocks is assumed. The local flow is governed by either Klinkenberg's law or Knudsen's diffusion law in the matrix while either a non-slip flow or a slip flow occurs in the fractures. Six new models are derived by homogenization, which are compared to the three models which were obtained for Darcy's regime in an earlier work. Each of these nine models is characterized by its macroscopic flow regime and by the type of macroscopic behavior it describes. Besides Darcy's and Klinkenberg's macroscopic flow regimes, a transition regime between Klinkenberg's and Knudsen's regimes is identified. The types of macroscopic behaviors include a dual and a single porosity description and an intermediate behavior that describes a single-porosity behavior, but in which the porosity of the entire fractured porous medium is accounted for.
\end{abstract}

Keywords Gas flow - Gas permeability - Klinkenberg's effect - Gas diffusion . Knudsen's diffusion - Fractured porous media - Dual-porosity - Continuum modeling . Homogenization

\footnotetext{
J. Chastanet

Environmental Engineering, Oregon State University,

Corvallis, OR 97331, USA

e-mail: chastaju@engr.orst.edu

P. Royer (ख) · J.-L. Auriault

Laboratoire "Sols, Solides, Structures" (3S), UJF, INPG, CNRS, BP 53,

38041 Grenoble Cedex, France

e-mail: pascale.royer@hmg.inpg.fr; jean-louis.auriault@hmg.inpg.fr
} 


\section{Introduction}

Darcy's law may break down for flows of gas at low pressure in porous media: in low permeable media, the gas permeability $K_{\mathrm{g}}$ of a porous sample can be greater than its liquid permeability $K_{1}$. This phenomenon is called Klinkenberg's effect and is described by the so-called Klinkenberg's law, which was first formulated by Adzumi (1937a, 1937b), later on by Klinkenberg (1941)

$$
K_{\mathrm{g}}=K_{1}\left(1+\frac{b}{p}\right),
$$

where $p$ is the gas pressure and $b>0$ is the Klinkenberg factor which depends on the medium properties, as well as to a lesser degree, on the nature of gas. Physically, Klinkenberg's effect is significant in any situation where the mean free path of gas molecules $\lambda$ is comparable with the pore size $l_{\mathrm{p}}$, which leads to a non-negligible Knudsen number $K n=\lambda / l_{\mathrm{p}} \approx 1$. Beyond, i.e at pressures for which the mean free path is much greater than the pore-size $(K n \gg 1)$, a diffusive transport proportional to the pressure gradient is observed. The phenomenon is called Knudsen diffusion. It has firstly been investigated by Knudsen (1909), who considered the flow of a highly rarefied gas flow in a cylindrical conduct. Later on, Derjaguin (1946) proposed the following transport equation for its description in a porous medium

$$
\vec{V}=-\frac{D_{\mathrm{g}}}{p} \vec{\nabla} \mathrm{p}
$$

where $\vec{V}$ denotes the gas filtration velocity and $D_{\mathrm{g}}$ represents the Knudsen diffusion coefficient, which strongly depends on pore geometry. For the flow of gas at low pressure in porous media, the flow regime is therefore conditioned by the Knudsen number and the regimes observed at the sample scale are the consequence of distinct physics of flow at the pore scale (Table 1$)$. Darcy's flow regime $(K n \ll 1)$ results from a pore-scale non-slip flow, described by the adherence condition on the fluid-solid boundary. When Klinkenberg's effect occurs, then significant molecular collisions are with the pore wall rather than with other gas molecules. Thus, the molecules on the fluid/solid boundary are no longer static and a wall-slip flow occurs: the local flow velocity is a non-vanishing velocity on the pore walls A tensorial form of Klinkenberg's law has been obtained by homogenization in (Skjetne and Auriault 1999), by starting from a local slip flow. When Knudsen's effect occurs, the gas is then so highly rarefied that molecule-molecule collisions become negligible with regard to molecule-wall collisions. As a result, gas flow at the pore scale can no longer be described by means of a continuum model. This case is beyond the scope of the present work, but we shall anyhow mention the work of Charrier and Dubroca (2003), who

Table 1 Gas flow regimes and their domains of validity

\begin{tabular}{llll}
\hline$K n$-values & $K n \ll 1$ & $K n \sim 1$ & $K n \gg 1$ \\
\hline $\begin{array}{l}\text { Sample scale regime } \\
\text { Pore scale regime }\end{array}$ & $\begin{array}{l}\text { Darcy's law } \\
\text { Stokes flow, wall } \\
\text { non-slip flow }\end{array}$ & $\begin{array}{l}\text { Klinkenberg's effect } \\
\text { Stokes flow, wall-slip flow }\end{array}$ & $\begin{array}{l}\text { Knudsen diffusion } \\
\text { Knudsen molecular flow, } \\
\text { no continuous description }\end{array}$ \\
\hline
\end{tabular}


uspcaled Boltzmann's equation to obtain a continuum macroscopic diffusion law. The present work is concerned with the occurrence of Klinkenberg's or Knudsen's effects in dual-porosity media, i.e. in media that consist of two interacting porous systems of distinctly different fluid transfer properties. The practical applications are important as accurate predictions of gas flow through dual-porosity media concern many areas: petroleum engineering, geothermal reservoir engineering, civil engineering and material engineering for the forming of paper or other fibrous materials. In the sequel, we consider the most widely used example of dual-porosity structure, namely that of a fractured porous medium. It is composed of an interconnected network of fractures and blocks of porous medium. Basically, the physics of flow in fractured porous media is such that the fluid preferentially flows in the fractures, while it is mainly stored in the porous blocks. In the study, we assume that a representative elementary volume (abbreviated r.e.v.) of the fractured porous medium can be defined. The objective is to derive mathematical models by upscaling the gas flow description at the r.e.v. scale. In the present work we consider either Klinkenberg's or Knudsen's local flow regimes in the porous matrix and we then compare the results with those obtained in Darcy's regime by (Royer and Auriault 1994). The method used for upscaling the local descriptions is the homogenization method for periodic structures-also called method of multiple scales - that has been introduced by (Bensoussan et al. 1978) and (Sanchez-Palencia 1980). As any continuum approach, the method of homogenization for periodic structures is based upon the fundamental assumption of separation of scales. The second assumption made is the periodicity of the medium. The advantage of the method is that, thanks to the periodicity, no preliminary assumption is required on the form of the macroscopic models. It should also be underlined that the periodicity has no impact on the form of the equivalent macroscopic models In the present study, we follow the methodology introduced by (Auriault 1991). The upscaling process consists of several steps from writing the physical description at the r.e.v. scale to finding the macroscopic model. The reader may refer to (Auriault 2005) for a detailed presentation of the methodology and to (Royer et al. 2002) for a brief introduction. Based on the use of dimensionless numbers, this approach provides a means for determining the domains of validity of derived models and is particularly well-adapted for the description of dual-porosity media (Auriault and Boutin 1992, 1993).

The paper is organized as follows. After describing the medium under consideration for the study, we then present the results obtained in Darcy's regime by (Royer and Auriault 1994). Next, we focus on the derivation of the macroscopic models in Klinkenberg's and Knudsen's regime. A first paragraph sets out to determine which local fracture flow regimes should be considered when either Klinkenberg's or Knudsen's effects occur in the porous matrix. The corresponding dimensionless local descriptions are then presented and a magnitude analysis of the dimensionless parameters is performed. This step allows pointing out any couplings that conduct to different macroscopic models as well as situations for which the macroscopic model does not exist. Application of the homogenization procedure leads to the macroscopic models which are presented and compared to the models obtained in Darcy's regime. Finally, we globally analyze the results obtained in Darcy's, Klinkenberg's and Knudsen's regimes by examining the types of macroscopic behaviors and the macroscopic flow regimes. 


\section{Medium under consideration}

As any continuum modeling approach, the method of multiple scales is based upon the fundamental assumption of separation of scales. Furthermore, the method also requires the condition of periodicity. We thus consider a periodic fractured porous medium of macroscopic characteristic size $L$. We further denote the period by $\Omega$, its scale length by $l$, and we formulate the condition of separation of scales as

$$
\varepsilon=\frac{l}{L} \ll 1
$$

Within the periodic cell, we denote by $\Omega_{\mathrm{f}}$ the fracture's domain, by $\Omega_{\mathrm{m}}$ the porous matrix domain and by $\Gamma$ their common boundary, as depicted in Fig. 1 . The fractures are assumed to be connected, whereas the connectivity of the microporous system is not required.

Using the two characteristic lengths, $l$ and $L$, and the physical space variable, $\vec{X}$, we define two dimensionless space variables: $\vec{y}=\vec{X}$ and $\vec{x}=\vec{X}$. If the condition of separation of scales is satisfied $(\varepsilon \ll 1)$, then $\vec{y}$ and $\vec{x}$ appear as two independent space variables: $\vec{y}$ is the microscopic space variable and describes the local scale, whereas $\vec{x}$ is the macroscopic variable. As a consequence, the unknown field quantities being considered (e.g. pressure, velocity, ...) are, a priori, functions of both space variables $\vec{y}$ and $\vec{x}$. Furthermore, invoking the differentiation rule of multiple variables, the gradient operator with respect to the physical space variable, $\vec{\nabla} X$, is written as

$$
\vec{\nabla} X=\frac{1}{l} \vec{\nabla}_{y}+\frac{1}{L} \vec{\nabla}_{x}
$$

where $\vec{\nabla}_{y}$ and $\vec{\nabla}_{x}$ are the gradient operators with respect to $\vec{y}$ and $\vec{x}$, respectively. As continuum models will be used to describe the fluid filtration in the porous matrix, we further assume that the pore-scale length, $l_{\mathrm{p}}$, is much smaller than the period size

$$
\alpha=\frac{l_{\mathrm{p}}}{l} \ll 1
$$

and we note

$$
\alpha=\mathrm{O}\left(\varepsilon^{n}\right) \quad \text { with } \quad n>0 .
$$

Parameter $n$ is a geometrical parameter of the medium: the greater the value of $n$, the smaller the micropore size.

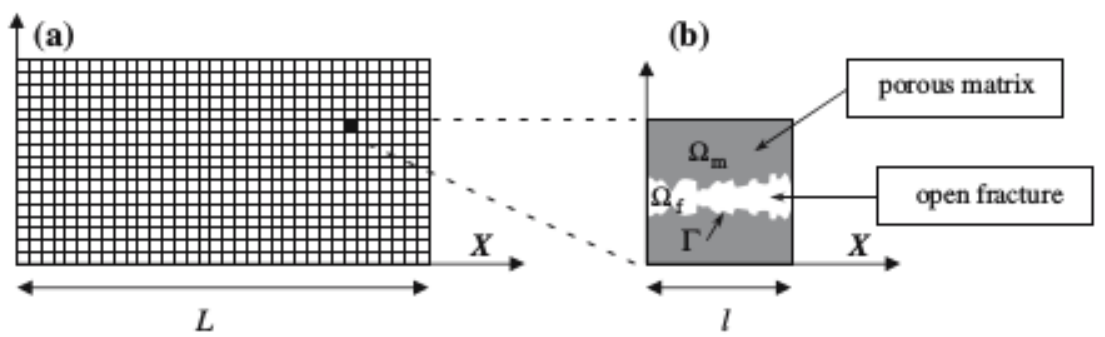

Fig. 1 A periodic fractured porous medium: (a) the macroscopic scale (of char acteristic length $L$ ), (b) the periodic cell (of characteristic length $l$ ) consists of a porous matrix domain and an open fracture domain 


\section{Results in Darcy's regime}

Royer and Auriault (1994) have considered gas flow in the above described medium, with Darcy's regime in the matrix and a non-slip flow in the fractures. For simplicity, a gas of linear equation of state has been considered: $p=A \rho$, where $p$ and $\rho$ denote the pressure and the density, respectively, and $A$ is a constant. By applying the homogenization theory, the three following first-order macroscopic models are obtained

$$
\begin{aligned}
& -\underline{\text { Model } \mathrm{I}_{1}}(n<1) \\
& \left(\phi_{\mathrm{f}}+\phi_{\mathrm{m}}\left(1-\phi_{\mathrm{f}}\right)\right) \frac{\partial p^{(0)}}{\partial t}-\vec{\nabla}_{x} \cdot\left(p^{(0)} \frac{\tilde{K}_{\mathrm{f}_{\mathrm{l}}}}{\mu} \vec{\nabla}_{x} p^{(0)}\right)=0,
\end{aligned}
$$

- $\underline{\text { Model } \mathrm{II}_{1}}(n=1)$

$$
\begin{aligned}
& \phi_{\mathrm{f}} \frac{\partial p_{\mathrm{f}}^{(0)}}{\partial t}+\phi_{\mathrm{m}} \frac{\partial\left\langle p_{\mathrm{m}}^{(0)}\right\rangle}{\partial t}-\vec{\nabla}_{x} \cdot\left(p_{\mathrm{f}}^{(0)} \frac{\tilde{K}_{\mathrm{f}_{\mathrm{l}}}}{\mu} \vec{\nabla}_{x} p_{\mathrm{f}}^{(0)}\right)=0, \\
& \qquad \frac{\text { Model III }}{1}(n>1) \\
& \phi_{\mathrm{f}} \frac{\partial p_{\mathrm{f}}^{(0)}}{\partial t}-\vec{\nabla}_{x} \cdot\left(p_{\mathrm{f}}^{(0)} \frac{\tilde{K}_{\mathrm{f}_{\mathrm{l}}}}{\mu} \vec{\nabla}_{x} p_{\mathrm{f}}^{(0)}\right)=0,
\end{aligned}
$$

where indexes ' $\mathrm{f}$ ' and ' $\mathrm{m}$ ' refer to the fracture and to the matrix, respectively, $\phi_{\mathrm{f}}$ and $\phi_{\mathrm{m}}$ denote the porosities and $\mu$ is the viscosity of gas. In these three models, the effective permeability is the fracture liquid permeability $\tilde{K}_{\mathrm{fl}}$. The domains of validity are defined by means of $n$, which indicates that the macroscopic behavior depends on the micropore size. The non-linearity of these three models stems from gas compressibility. We firstly note that model $\mathrm{III}_{1}$ describes the flow in a fractured non-porous medium and is ther efore a single-porosity model. The notable property of Model $\mathrm{II}_{1}$ is that it is a two-pressure-field model $\left(p_{\mathrm{m}}^{(0)} \neq p_{\mathrm{f}}^{(0)}\right)$. The $\Omega$-periodic matrix pressure field $p_{\mathrm{m}}^{(0)}$ satisfies the boundary-value problem that describes the local matrix flow regime

$$
\begin{aligned}
& \phi_{\mathrm{m}} \frac{\partial p_{\mathrm{m}}^{(0)}}{\partial t}-\vec{\nabla}_{y} \cdot\left(p_{\mathrm{m}}^{(0)} \frac{K_{\mathrm{m}_{\mathrm{l}}}}{\mu} \vec{\nabla}_{y} p_{\mathrm{m}}^{(0)}\right)=0 \text { in } \Omega_{\mathrm{m}}, \\
& p_{\mathrm{m}}^{(0)}=p_{\mathrm{f}}^{(0)} \text { on } \Gamma,
\end{aligned}
$$

where $K_{\mathrm{m}}$ is the intrinsic permeability of the matrix. The average $\left\langle p_{\mathrm{m}}^{(0)}\right\rangle$ is defined by

$$
\left\langle p_{\mathrm{m}}^{(0)}\right\rangle=\frac{1}{|\Omega|} \int_{\Omega_{\mathrm{m}}} p_{\mathrm{m}}^{(0)} d \Omega .
$$

Model $\mathrm{II}_{1}$ describes a dual-porosity behavior: it characterizes the case of coupled matrix and fracture flows. Both pressure fields are actually such that $p_{\mathrm{m}}^{(0)}=\mathcal{F}\left(p_{\mathrm{f}}^{(0)}\right)$, where $\mathcal{F}$ is a non-linear functional with memory effects. Because of the non-linearities that stem from gas compressibility, there is no general analytical expression for functional $\mathcal{F}$. It should also be underlined that model $\mathrm{I}_{1}$ cannot be expressed as a twoequation model including a matrix-fracture exchange term as in the pioneering work of (Barenblatt et al. 1960) on dual-porosity modeling. When considering a slightly compressible fluid, a linearized form of model $\mathrm{II}_{1}$ is obtained (Royer and Auriault 
1994). An analytical expression of $\mathcal{F}$ can then be determined. A model of Barenblatt's type can then provide an approximation in quasi-steady state regime. This latter issue is also addressed in (Moyne 1997). Finally, in model $I_{1}$, the pressure fields are equal: $p^{(0)}=p_{\mathrm{f}}^{(0)}=p_{\mathrm{m}}^{(0)}$. This is a single-porosity model, but in which the effective permeability is the fracture permeability, while the effective porosity is that of the whole fractured porous medium. We designate this property by "reservoir effect" to signify that the porous matrix domain plays the role of a gas reservoir in that case. The present work is aimed at replacing Darcy's law at the local scale by Klinkenberg's or Knudsen's law and then at examining the impact on the above presented models.

\section{Derivation of macroscopic models in Klinkenberg's and Knudsen's regimes}

In this section we present the detailed preliminary local-scale analysis required for applying the homogenization procedure. We then present the derived macroscopic models and compare them to those obtained in Darcy's regime.

\subsection{Analysis of local gas flow regimes}

The first step consists in determining which types of flows may occur in the fractures when either Klinkenberg's or Knudsen's effects are considered in the porous matrix. We firstly determine the conditions under which Klinkenberg's and Knudsen effects occur in the porous matrix. We then list the appropriate flow regimes in the fractures. Finally, we identify which fracture flow regimes we consider for each matrix flow.

\subsubsection{Flow regimes in the matrix}

Gas flow regime in the porous matrix is conditioned by the order of magnitude of the pore-scale Knudsen number, defined by

$$
K n_{\mathrm{p}}=\frac{\lambda_{\mathrm{p}}}{l_{\mathrm{p}}}
$$

in which $\lambda_{\mathrm{p}}$ denotes the mean free path at the pore scale. As $K n_{\mathrm{p}}$ is a quantity defined at the pore scale, the appropriate scale-ratio for estimating its order of magnitude is $\alpha$, and we put

$$
K n_{\mathrm{p}}=\mathcal{O}\left(\alpha^{q}\right) .
$$

In Klinkenberg's regime, the gas permeability $k_{\mathrm{m}_{\mathrm{g}}}$ satisfies Klinkenberg's law, which can be expressed as follows (Klinkenberg 1941):

$$
k_{\mathrm{m}_{\mathrm{g}}}=k_{\mathrm{m}_{\mathrm{l}}}\left(1+4 c_{\mathrm{m}} K n_{\mathrm{p}}\right) \text {, }
$$

where $k_{\mathrm{m}_{\mathrm{l}}}$ denotes the matrix liquid permeability and $c_{\mathrm{m}}$ is a constant whose value is close to 1 . From the above expression, it follows that Klinkenberg's effect is significant within the matrix when $4 c_{\mathrm{m}} K n_{\mathrm{p}}>1$, i.e. when $K n_{\mathrm{p}}>\mathcal{O}(1)$. As for Knudsen's diffusion, it will occur provided that $K n_{\mathrm{p}} \gg 1$. From the above argument we thus conclude that the filtration law in the porous matrix is

$$
\left\{\begin{array}{l}
\bullet \text { Klinkenberg's law when } \mathcal{O}\left(\alpha^{0}\right)<K n_{\mathrm{p}}<\mathcal{O}\left(\alpha^{-1}\right), \\
\bullet \text { Knudsen's diffusion law when } K n_{\mathrm{p}} \geq \mathcal{O}\left(\alpha^{-1}\right) .
\end{array}\right.
$$




\subsubsection{Flow regimes in the fractures}

Let us now analyze the distinct flow regimes that may occur in the fractures. Likewise, gas flow regime in the fractures is conditioned by the magnitude of the fracture-scale Knudsen number

$$
K n_{\mathrm{f}}=\frac{\lambda_{\mathrm{f}}}{l},
$$

where $\lambda_{\mathrm{f}}$ represents the mean free path of gas molecules in the fractures. As it is defined at the period's scale, $K n_{\mathrm{f}}$ must be evaluated by means of the scale-ratio $\varepsilon$. The possible fracture flow regimes are the following

$$
\left\{\begin{array}{l}
\bullet \text { Non-slip flow when } K n_{\mathrm{f}} \leq \mathcal{O}(\varepsilon), \\
\bullet \text { Slip flow when } \mathcal{O}(\varepsilon) \ll K n_{\mathrm{f}} \leq \mathcal{O}\left(\varepsilon^{0}\right), \\
\bullet \text { Knudsen's molecular flow when } K n_{\mathrm{f}}>\mathcal{O}\left(\varepsilon^{0}\right) \text {. }
\end{array}\right.
$$

As there is no continuous pore-scale model for describing a Knudsen's molecular flow, we restrict the study to the case

$$
K n_{\mathrm{f}} \leq \mathcal{O}\left(\varepsilon^{0}\right)
$$

Now, the magnitudes of both Knudsen numbers are actually linked. Because it can be assumed that the pressure fields in both domains are of same magnitude, we may consider that: $\lambda_{p}=\mathcal{O}\left(\lambda_{f}\right)$. Then, from Equations (4.1) and (4.5), we get

$$
K n_{\mathrm{f}}=\mathcal{O}\left(K n_{\mathrm{p}} \alpha\right)
$$

which from (4.2) and (2.4) leads to

$$
K n_{\mathrm{f}}=\mathcal{O}\left(\varepsilon^{n(q+1)}\right) .
$$

Therefore, the fracture flow regimes under consideration in the study are the following:

$$
\left\{\begin{array}{l}
\bullet \text { Non-slip flow when } n \geq \frac{1}{q+1}, \\
\bullet \text { Slip flow } \quad \text { when } 0 \leq n(q+1)<1
\end{array}\right.
$$

\subsubsection{Possible combinations between matrix and fracture flow regimes}

We are now in position to determine which types of flows we may consider in the fractures, when either Klinkenberg's or Knudsen's effects occur in the porous matrix. Firstly, from definition (4.9) of $K n_{\mathrm{f}}$ magnitude and furthermore because $n>0$, it turns out that the restriction (4.7) on the order of $K n_{\mathrm{f}}$ entails that $K n_{\mathrm{p}} \leq \mathcal{O}\left(\alpha^{-1}\right)$, which translates into the condition $q \geq-1$. The magnitudes of $K n_{\mathrm{p}}$ under consideration in the study are therefore the following:

$$
K n_{\mathrm{p}}=\mathcal{O}\left(\alpha^{q}\right) \quad \text { with }-1 \leq q<0,
$$

and the matrix filtration law is

$$
\left\{\begin{array}{l}
\bullet \text { Klinkenberg's law when }-1<q<0, \\
\bullet \text { Knudsen's diffusion law when } q=-1
\end{array}\right.
$$


From (4.12) and (4.10), it follows that when Klinkenberg's effect occurs in the matrix $(-1<q<0)$, the fracture flow is either a non-slip flow $(n \geq 1 / q+1)$, or a slip flow ( $n<1 / q+1)$. When Knudsen's diffusion occurs in the matrix $(q=-1)$, then $K n_{\mathrm{f}}=\mathcal{O}\left(\varepsilon^{0}\right)$ and a slip flow necessarily takes place in the fractures. Therefore, the flow regime analysis branches off into the three following situations, which are summed up on a $K n_{\mathrm{p}}-\alpha$ diagram in Fig. 2.

$$
\left\{\begin{aligned}
& \text { Situation } \mathcal{A}:\left(-1<q<0 \text { and } n \geq \frac{1}{q+1}\right) \\
& \text { Klinkenberg's law in the porous matrix } \\
& \text { Non-slip flow in the fracture } \\
& \text { Situation } \mathcal{B}:\left(-1<q<0 \text { and } n<\frac{1}{q+1}\right) \\
& \text { Klinkenberg's law in the porous matrix } \\
& \text { Slip flow in the fracture } \\
& \text { Situation } \mathcal{C}:(q=-1) \\
& \text { Knudsen's diffusion law in the porous matrix } \\
& \text { Slip flow in the fracture }
\end{aligned}\right.
$$

Our objective is thus to determine the macroscopic models in the three above defined situations and to compare the results with those obtained in Darcy's regime by Royer and Auriault (1994), presented in Section 3.

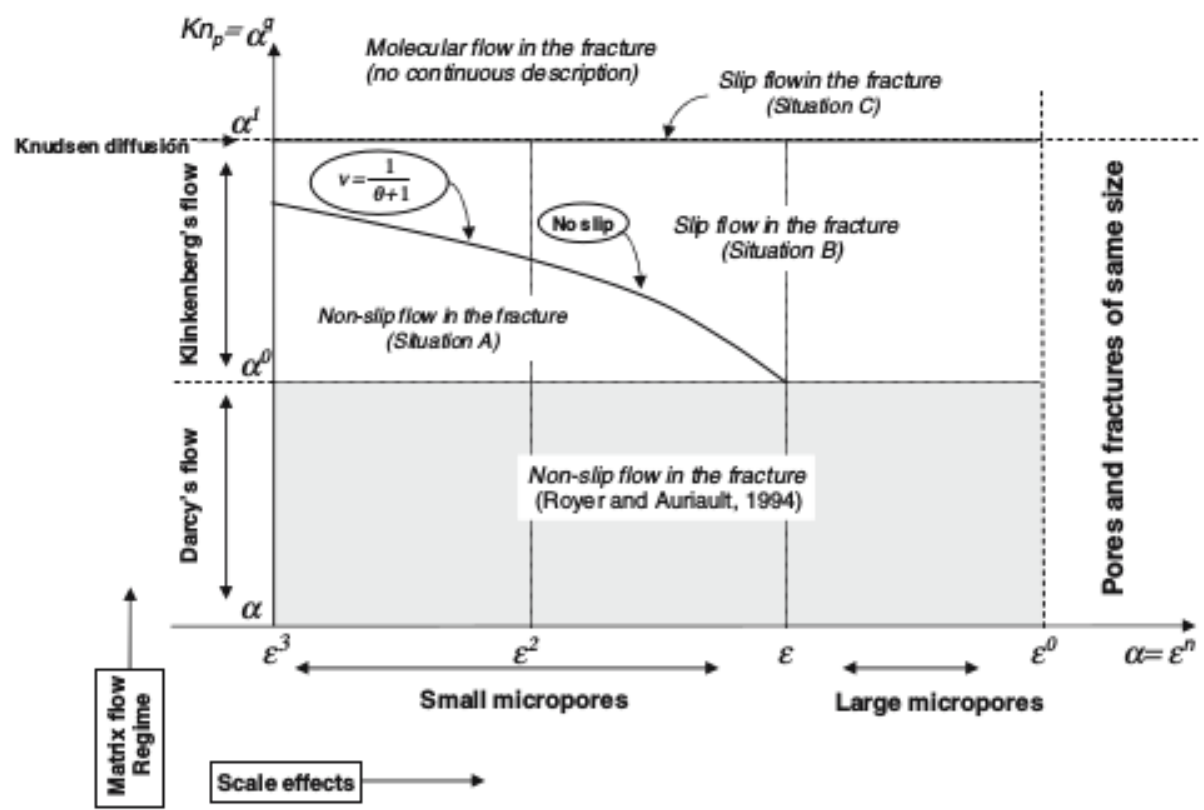

Fig. $2 K n_{\mathrm{p}}-\alpha$ diagram of local flow regimes 


\subsubsection{Introduction}

A local description is the set of the equations that describe the flow within the period depicted in Section 2. It therefore consists of the flow equations in the porous matrix, in the fractures and on the matrix/fractures interface. The first step of the homogenization methodology being used (Auriault 1991) is to write the local description under consideration in a dimensionless form. Let denote by $\Phi$ and by $\xi$ the dimensionless counterpart and the characteristic value, respectively, of a quantity $\Psi$; $\Phi$ is given by $\Phi=\Psi / \xi$. Then, replacing $\Psi$ by $\xi \Phi$ in the dimensional local description leads to its dimensionless writing. Dimensionless numbers thus appear in the dimensionless description, which are defined by means of characteristic values. Below, we present the three dimensionless local descriptions that describe situations $\mathcal{A}, \mathcal{B}$ and $\mathcal{C}$. Any dimensionless writing of equations requires the arbitrary choice of characteristic length and time, for the definition of dimensionless space and time derivatives, respectively. We choose the macroscopic characteristic length $L$ and the macroscopic characteristic time of flow, $T$. With regard to (2.2), the dimensionless gradient operator, $\vec{\nabla}$, is thus given by

$$
\vec{\nabla}=L \vec{\nabla} x=\varepsilon^{-1} \vec{\nabla}_{y}+\vec{\nabla} x
$$

As in (Royer and Auriault 1994), we consider a gas of linear equation of state, i.e. such that $p=A \rho$, where $p$ and $\rho$ denote the pressure and the density, respectively, and where $A$ is a constant.

\subsubsection{In the porous matrix $\left(\Omega_{\mathrm{m}}\right)$}

In the porous matrix, gas flow is governed by a filtration law and a mass-balance equation. When $-1<q<0$, gas filtration is governed by Klinkenberg's law. In dimensional form it reads

$$
\vec{V}_{\mathrm{m}}=-\frac{k_{\mathrm{m}_{\mathrm{g}}}}{\mu_{0}} \vec{\nabla}_{X} P_{\mathrm{m}}
$$

in which $\vec{V}_{\mathrm{m}}$ and $P_{\mathrm{m}}$ are the physical fields of velocity and pressure and $\mu_{0}$ represents the viscosity. The gas permeability $k_{\mathrm{m}_{\mathrm{g}}}$ satisfies Klinkenberg's law (4.3). As $k_{\mathrm{m}_{\mathrm{l}}}$ is of magnitude $l_{\mathrm{p}}^{2}$, we deduce from (4.3) that a characteristic value of $k_{\mathrm{m}_{\mathrm{g}}}$ is given by $K_{\mathrm{m}_{\mathrm{c}}}=K n_{\mathrm{p}} l_{\mathrm{p}}^{2}$. The dimensionless writing of Equation (4.15) is therefore given by

$$
\vec{v}_{\mathrm{m}}=-\mathcal{Q}_{\mathrm{m}} \frac{K_{\mathrm{m}_{\mathrm{g}}}}{\mu} \vec{\nabla} p_{\mathrm{m}}, \quad \mathcal{Q}_{\mathrm{m}}=\mathcal{O}\left(\frac{l_{\mathrm{p}}^{2}}{\mu_{\mathrm{c}}} K n_{\mathrm{p}} \frac{\delta P}{L V_{\mathrm{m}}}\right)
$$

in which $\vec{v}_{\mathrm{m}}=\vec{V}_{\mathrm{m}} / V_{\mathrm{m}}, K_{\mathrm{m}_{\mathrm{g}}}=k_{\mathrm{m}_{\mathrm{g}}} / K_{\mathrm{m}_{\mathrm{gc}}}, \mu=\mu_{0} / \mu_{\mathrm{c}}$ and $p_{\mathrm{m}}=P_{\mathrm{m}} / \delta P$ are the dimensionless counterparts of $\vec{V}_{\mathrm{m}}, k_{\mathrm{m}_{\mathrm{g}}}, \mu_{0}$ and $P_{\mathrm{m}}$, respectively, and where $V_{\mathrm{m}}$ represents the characteristic velocity in the porous matrix, $\mu_{\mathrm{c}}$ is the characteristic value of the viscosity, and $\delta P$ denotes the characteristic macroscopic pressure drop. When $q=-1$, gas flow is described by Knudsen's diffusion law, which in dimensional form is expressed as

$$
\vec{V}_{\mathrm{m}}=-\frac{d_{\mathrm{m}}}{P_{\mathrm{m}}} \vec{\nabla}_{X} P_{\mathrm{m}}
$$


The Knudsen diffusion coefficient $d_{\mathrm{m}_{\mathrm{g}}}$ can be defined by (Kast and Hohenthanner 2000) $d_{\mathrm{m}_{\mathrm{g}}}=l_{\mathrm{p}} \phi_{\mathrm{m}} / 3 \tau_{\mathrm{m}} \sqrt{8 R t_{\mathrm{m}} / \pi M}$, where $\tau_{\mathrm{m}}$ denotes the Knudsen tortuosity, $R$ is the gas constant, $t_{\mathrm{m}}$ is the temperature and $M$ is the molecular weight. By considering the definition of the mean free path given in (Cercignani, 1988), $\lambda_{\mathrm{p}}=$ $\mu_{0} / P_{\mathrm{m}} \sqrt{\pi R t_{\mathrm{m}} / 2 M}$, we get the following characteristic value for the Knudsen coefficient: $D_{m_{\mathrm{gc}}}=\mathcal{O}\left(P_{\mathrm{m}} l_{\mathrm{p}} \lambda_{\mathrm{p}} / \mu_{0}\right)$. Consequently, the dimensionless Knudsen's law reads

$$
\vec{v}_{\mathrm{m}}=-\mathcal{Q}_{\mathrm{m}} \frac{D_{\mathrm{m}_{\mathrm{g}}}}{p_{\mathrm{m}}} \vec{\nabla} p_{\mathrm{m}}, \quad \mathcal{Q}_{\mathrm{m}}=\mathcal{O}\left(\frac{l_{\mathrm{p}}^{2}}{\mu_{\mathrm{c}}} K n_{\mathrm{p}} \frac{\delta P}{L V_{\mathrm{m}}}\right)
$$

in which $D_{\mathrm{m}_{\mathrm{g}}}=d_{\mathrm{m}_{\mathrm{g}}} / D_{m_{\mathrm{g}_{\mathrm{c}}}}$ denotes the dimensionless Knudsen coefficient. In other words, dimensionless number $\mathcal{Q}_{\mathrm{m}}$ arises in both dimensionless filtration laws (4.16) and (4.18). Finally, we may express the filtration law in the porous matrix by

$$
\vec{v}_{\mathrm{m}}=-\mathcal{Q}_{\mathrm{m}} B_{\mathrm{m}} \vec{\nabla} p_{\mathrm{m}}, \quad \mathcal{Q}_{\mathrm{m}}=\mathcal{O}\left(\frac{l_{\mathrm{p}}^{2}}{\mu_{\mathrm{c}}} K n_{\mathrm{p}} \frac{\delta P}{L V_{\mathrm{m}}}\right),
$$

where

$$
B_{\mathrm{m}}=\left\{\begin{array}{l}
\frac{K_{\mathrm{m}_{\mathrm{g}}}}{\mu} \text { when }-1<q<0 \text { (Klinkenberg's law), } \\
\frac{D_{\mathrm{m}_{\mathrm{g}}}}{p_{\mathrm{m}}} \text { when } q=-1 \text { (Knudsen's law). }
\end{array}\right.
$$

The description in the porous matrix must be completed by the dimensionless massbalance equation, which reads

$$
\mathcal{S}_{\mathrm{m}} \phi_{\mathrm{m}} \frac{\partial p_{\mathrm{m}}}{\partial t}+\vec{\nabla} \cdot\left(p_{\mathrm{m}} \vec{v}_{\mathrm{m}}\right)=0, \quad \mathcal{S}_{\mathrm{m}}=\mathcal{O}\left(\frac{L}{T V_{\mathrm{m}}}\right) .
$$

\subsubsection{In the fractures $\left(\Omega_{\mathrm{f}}\right)$}

Gas flow in the fractures is governed by Stokes equation and the mass-balance equation. When placed in dimensionless form, they read

$$
\begin{aligned}
& \mu \vec{\nabla}^{2} \vec{v}_{\mathrm{f}}+(v+\mu) \vec{\nabla}\left(\vec{\nabla} \cdot \vec{v}_{\mathrm{f}}\right)-\mathcal{Q}_{\mathrm{f}} \vec{\nabla} p_{\mathrm{f}}=\overrightarrow{0}, \\
& \mathcal{Q}_{\mathrm{f}}=\mathcal{O}\left(\frac{l^{2}}{\mu_{\mathrm{c}}} \frac{\delta P}{L V_{\mathrm{f}}}\right), \\
& \mathcal{S}_{\mathrm{f}} \frac{\partial p_{\mathrm{f}}}{\partial t}+\vec{\nabla} \cdot\left(p_{\mathrm{f}} \vec{v}_{\mathrm{f}}\right)=0, \quad \mathcal{S}_{\mathrm{f}}=\mathcal{O}\left(\frac{L}{T V_{\mathrm{f}}}\right),
\end{aligned}
$$

where $\vec{v}_{\mathrm{f}}$ and $p_{\mathrm{f}}$ denote the dimensionless fields of velocity and pressure in the fracture domain, respectively, and where $V_{\mathrm{f}}$ is a characteristic value of fracture flow velocity.

\subsubsection{Over the matrix/fracture interface $(\Gamma)$}

The conditions on $\Gamma$ express the continuity of pressures and of velocities In dimensionless form, the continuity of pressures simply reads

$$
p_{\mathrm{f}}=p_{\mathrm{m}} .
$$


The continuity of velocities depends on the flow regime being considered in the fractures. When a non-slip flow occurs in the fractures $(n \geq 1 / q+1)$, the dimensionless continuity of velocities is written as

$$
\vec{v}_{\mathrm{f}}=\gamma \vec{v}_{\mathrm{m}}, \quad \gamma=\mathcal{O}\left(\frac{V_{\mathrm{m}}}{V_{\mathrm{f}}}\right) .
$$

When a slip flow occurs in the fractures ( $n<1 / q+1$ or $q=-1$ ), we consider the slippage law used in (Skjetne and Auriault 1999), but adapted to the case of a dual-porosity medium. In dimensional form, it reads

$$
\vec{V}_{\mathrm{f}}=\vec{V}_{\mathrm{m}}-c_{\mathrm{f}} \lambda_{\mathrm{f}}\left(\vec{t}_{1} \cdot \vec{\nabla}_{X} \vec{V}_{\mathrm{f}} \cdot \vec{n}\right) \vec{t}_{1}
$$

where $\vec{t}_{1}$ and $\vec{n}$ are the unit tangential and normal vectors associated with the velocity and with the interface, and where $c_{\mathrm{f}}$ is a constant whose value is close to 1 . By noticing that $c_{\mathrm{f}} \lambda_{\mathrm{f}} / L=\mathcal{O}\left(\varepsilon K n_{\mathrm{f}}\right)$, we get the following dimensionless writing

$$
\vec{v}_{\mathrm{f}}=\gamma \vec{v}_{\mathrm{m}}-\varepsilon K n_{\mathrm{f}}\left(\vec{t}_{1} \cdot \vec{\nabla} \vec{v}_{\mathrm{f}} \cdot \vec{n}\right) \vec{t}_{1} .
$$

Dimensionless equations (4.25) and (4.27) can be expressed by the following single equation

$$
\vec{v}_{\mathrm{f}}=\gamma \vec{v}_{\mathrm{m}}-\varepsilon K n_{\mathrm{f}} \vec{v}_{\mathrm{f}_{\Gamma}}, \quad \gamma=\mathcal{O}\left(\frac{V_{\mathrm{m}}}{V_{\mathrm{f}}}\right),
$$

where the slippage velocity $\vec{v}_{\mathrm{f}_{\Gamma}}$ is such that

$$
\vec{v}_{\mathrm{f}_{\Gamma}}=\left\{\begin{array}{l}
\overrightarrow{0} \text { when } n \geq 1 / q+1 \text { (non-slip flow), } \\
\left(\overrightarrow{t_{1}} \cdot \vec{\nabla} \vec{v}_{\mathrm{f}} \cdot \vec{n}\right) \vec{t}_{1} \text { when } n<1 / q+1 \text { or } q=-1 \text { (slip flow). }
\end{array}\right.
$$

\subsection{Estimation of dimensionless numbers}

The methodology introduced in (Auriault 1991) consists then in estimating all dimensionless numbers with respect to the small parameter $\varepsilon$. This step allows identification of the distinct dimensionless local descriptions, the upscaling of which may lead to distinct macroscopic models. A dimensionless number $Q$ is said to be order of $\varepsilon^{p}$ when

$$
\varepsilon^{p+1} \ll Q \ll \varepsilon^{p-1} .
$$

Firstly, noticing that Stokes equation in the fracture indicates that the local flow is generated by a macroscopic pressure gradient we deduce $\frac{\mu V_{f}}{l^{2}}=\mathcal{O}\left(\frac{\delta P}{L}\right)$, from which we get

$$
\mathcal{Q}_{\mathrm{f}}=\mathcal{O}\left(\varepsilon^{-2}\right), \quad \mathcal{Q}_{\mathrm{m}}=\mathcal{O}\left(\varepsilon^{-2} \alpha^{2} K n_{\mathrm{p}} \gamma^{-1}\right) .
$$

Now, let consider the case of a transient macroscopic regime. In an order-of-magnitude sense and due to fracture flow predominance, this is expressed by $T=\frac{L}{V_{\mathrm{f}}}$. Therefore, both Strouhal numbers are such that

$$
\mathcal{S}_{\mathrm{f}}=\mathcal{O}(1), \quad \mathcal{S}_{\mathrm{m}}=\mathcal{O}\left(\gamma^{-1}\right) .
$$

The remaining parameter to be estimated is the velocity ratio, $\gamma$. This parameter may actually take several orders of magnitude. Its determination requires a specific 
analysis, presented in Appendix A. We obtain

$$
\gamma=\mathcal{O}\left(\frac{V_{\mathrm{m}}}{V_{\mathrm{f}}}\right)= \begin{cases}\mathcal{O}(\varepsilon) & \text { if } n \leq \frac{2}{q+2}, \\ \mathcal{O}(\varepsilon) & \text { if } n=\frac{2}{q+2}, \\ \mathcal{O}\left(K n_{\mathrm{p}} \alpha^{2} \varepsilon^{-1}\right) & \text { if } n \geq \frac{2}{q+2} .\end{cases}
$$

4.4 Homogenization procedure

According to the above estimates, the dimensionless local description that consists of equations (4.19), (4.21), (4.22), (4.23), (4.24) and (4.28) may now be written as

$$
\begin{aligned}
& \vec{v}_{\mathrm{m}}=-\varepsilon^{n(q+2)-2} \gamma^{-1} B_{\mathrm{m}} \vec{\nabla} p_{\mathrm{m}} \quad \text { in } \Omega_{\mathrm{m}}, \\
& \gamma^{-1} \phi_{\mathrm{m}} \frac{\partial p_{\mathrm{m}}}{\partial t}+\vec{\nabla} \cdot\left(p_{\mathrm{m}} \vec{v}_{\mathrm{m}}\right)=0 \quad \text { in } \Omega_{\mathrm{m}}, \\
& \mu \vec{\nabla}^{2} \vec{v}_{\mathrm{f}}+(v+\mu) \vec{\nabla}\left(\vec{\nabla} \cdot \vec{v}_{\mathrm{f}}\right)-\varepsilon^{-2} \vec{\nabla} p_{\mathrm{f}}=\overrightarrow{0} \quad \text { in } \Omega_{\mathrm{f}}, \\
& \frac{\partial p_{\mathrm{f}}}{\partial t}+\vec{\nabla} \cdot\left(p_{\mathrm{f}} \vec{v}_{\mathrm{f}}\right)=0 \quad \text { in } \Omega_{\mathrm{f}}, \\
& p_{\mathrm{f}}=p_{\mathrm{m}} \quad \text { on } \Gamma, \\
& \vec{v}_{\mathrm{f}}=\gamma \vec{v}_{\mathrm{m}}-\varepsilon K n_{\mathrm{f}} \vec{v}_{\mathrm{f}_{\Gamma}} \quad \text { on } \Gamma
\end{aligned}
$$

in which the dimensionless gradient operator $\vec{\nabla}$ satisfies (4.14), $q$ is such that $-1 \leq$ $q<0$, and where $B_{\mathrm{m}}, \gamma$ and $\vec{v}_{\mathrm{f}_{\Gamma}}$ are defined by (4.20), (4.33) and (4.29), respectively. We shall now successively consider situations $\mathcal{A}, \mathcal{B}$ and $\mathcal{C}$, defined by (4.13). In each situation, we examine the three possible orders for $\gamma$ (4.33). We then apply the homogenization procedure to each local description being thus determined. It is based on the fundamental statement that as the scales are well separated $(\varepsilon \ll 1)$, then the physical variables $\left(\vec{v}_{\mathrm{m}}, p_{\mathrm{m}}, \vec{v}_{\mathrm{f}}, p_{\mathrm{f}}, K_{\mathrm{m}_{\mathrm{g}}}, \vec{t}_{1}\right)$ can be looked for in the form of asymptotic expansions in powers of $\varepsilon$ :

$$
\phi(\vec{y}, \vec{x})=\phi^{(0)}(\vec{y}, \vec{x})+\varepsilon \phi^{(1)}(\vec{y}, \vec{x})+\varepsilon^{2} \phi^{(2)}(\vec{y}, \vec{x})+\cdots
$$

in which functions $\phi^{(i)}$ are $\vec{y}$-periodic and dimensionless. The method consists in incorporating these asymptotic expansions in the dimensionless local description. This leads to approximate governing equations and boundary-conditions at the successive orders, which together with the condition of periodicity define boundary-value problems in the periodic cell. Existence of solutions requires that volume averaged equations be satisfied. These latter actually describe the macroscopic behavior at successive orders of $\varepsilon$.

Below, we present the macroscopic models obtained in situations $\mathcal{A}, \mathcal{B}$ and $\mathcal{C}$, defined by (4.13). Details on the derivation of one model are given in Appendix B.

\subsection{Macroscopic models}

\subsubsection{Klinkenberg's law in the matrix and no-slip flow in the fracture (situation $\mathcal{A})$}

It can easily be shown that when $-1<q<0$ and $n \geq 1 / q+1$, we necessarily have $n>2 / q+2$, which according to (4.33) indicates that only one order of magnitude for the velocity ratio is possible. The corresponding derived macroscopic model is identical to single-porosity model $\mathrm{II}_{1}$ (3.3). As the effective permeability is the liquid 
permeability $\tilde{K}_{\mathrm{f}_{\mathrm{l}}}$, we thus conclude that Klinkenberg's effect in the porous matrix at the local scale has no influence on the macroscopic flow regime in that case.

\subsubsection{Klinkenberg's law in the matrix and slip flow in the fracture (situation $\mathcal{B}$ )}

When $-1<q<0$ and $n<\frac{1}{q+1}$, the three possible orders for $\gamma$ (4.33) lead to the three following first-order macroscopic models

$$
\begin{aligned}
& \qquad \underline{\text { Model } \mathrm{I}_{\mathrm{g}}}\left(n<\frac{2}{q+2}\right) \\
& \left(\phi_{\mathrm{f}}+\phi_{\mathrm{m}}\left(1-\phi_{\mathrm{f}}\right)\right) \frac{\partial p^{(0)}}{\partial t}-\vec{\nabla}_{x} \cdot\left(p^{(0)} \frac{\tilde{K}_{\mathrm{f}_{\mathrm{g}}}}{\mu} \vec{\nabla}_{x} p^{(0)}\right)=0, \\
& \bullet \frac{\operatorname{Model~II} \mathrm{g}}{}\left(n=\frac{2}{q+2}\right) \\
& \phi_{\mathrm{f}} \frac{\partial p_{\mathrm{f}}^{(0)}}{\partial t}+\phi_{\mathrm{m}} \frac{\partial\left\langle p_{\mathrm{m}}^{(0)}\right\rangle}{\partial t}-\vec{\nabla}_{x} \cdot\left(p_{\mathrm{f}}^{(0)} \frac{\tilde{K}_{\mathrm{f}}}{\mu} \vec{\nabla}_{x} p_{\mathrm{f}}^{(0)}\right)=0, \\
& \bullet \frac{\operatorname{Model~III~}_{\mathrm{g}}}{}\left(n>\frac{2}{q+2}\right) \\
& \phi_{\mathrm{f}} \frac{\partial p_{\mathrm{f}}^{(0)}}{\partial t}-\vec{\nabla}_{x} \cdot\left(p_{\mathrm{f}}^{(0)} \frac{\tilde{K}_{\mathrm{f}_{\mathrm{g}}}}{\mu} \vec{\nabla}_{x} p_{\mathrm{f}}^{(0)}\right)=0 .
\end{aligned}
$$

In model $\mathrm{II}_{\mathrm{g}}, p_{\mathrm{m}}^{(0)}$ is the $\Omega$-periodic solution to

$$
\begin{aligned}
& \phi_{\mathrm{m}} \frac{\partial p_{\mathrm{m}}^{(0)}}{\partial t}-\vec{\nabla}_{y} \cdot\left(p_{\mathrm{m}}^{(0)} \frac{K_{\mathrm{m}}}{\mu} \vec{\nabla}_{y} p_{\mathrm{m}}^{(0)}\right)=0 \text { in } \Omega_{\mathrm{m}}, \\
& p_{\mathrm{m}}^{(0)}=p_{\mathrm{f}}^{(0)} \quad \text { on } \Gamma .
\end{aligned}
$$

Details on the derivation of model $\mathrm{I}_{\mathrm{g}}$ are given in Appendix B. We note that the three models are of same types as those derived in Darcy's regime (see Section 3), with the difference that in the present situation, the effective permeability is the effective fracture gas permeability, $\tilde{K}_{\mathrm{f}_{\mathrm{g}}}$ which satisfies

$$
\tilde{K}_{\mathrm{f}_{\mathrm{g}}}=\tilde{K}_{\mathrm{f}_{1}}\left(\tilde{I}+K n_{\mathrm{f}} \tilde{C}\right) \text {, }
$$

where $\tilde{C}$ is a positive tensor (Skjetne and Auriault 1999). Equation (4.44) is the tensorial form of Klinkenberg's law. Details about its derivation are given in Appendix B and in (Skjetne and Auriault 1999). The exact definition of $\tilde{K}_{\mathrm{f}_{g}}$ is given in Appendix C. A macroscopic Klinkenberg's effect therefore occurs in that case and the Klinkenberg's gas permeability constitutes an additional non-linearity in the models. We note that the domains of validity are defined by means of $n$ and $q$. Therefore, when Klinkenberg's effect occurs locally in the porous matrix, a macroscopic Klinkenberg's effect appears at the macroscopic scale and the type of behavior (reservoir effect, dual-porosity or single-porosity) is subordinate to both the micropore size and the value of the Knudsen number. 
When $q=-1$, the three cases defined by (4.33) lead to the three following first-order macroscopic models

$$
\begin{aligned}
& \text { - Model } \mathrm{I}_{\mathrm{g}}^{*}(n<2) \\
& \left(\phi_{\mathrm{f}}+\phi_{\mathrm{m}}\left(1-\phi_{\mathrm{f}}\right)\right) \frac{\partial p^{(0)}}{\partial t}-\vec{\nabla}_{x} \cdot\left(p^{(0)} \frac{\tilde{K}_{\mathrm{f}_{\mathrm{g}}}^{*}}{\mu} \vec{\nabla}_{x} p^{(0)}\right)=0 \text {, } \\
& \text { - } \underline{\text { Model } \mathrm{II}_{\mathrm{g}}^{*}(n=2)} \\
& \phi_{\mathrm{f}} \frac{\partial p_{\mathrm{f}}^{(0)}}{\partial t}+\phi_{\mathrm{m}} \frac{\partial\left\langle p_{\mathrm{m}}^{(0)}\right\rangle}{\partial t}-\vec{\nabla}_{x} \cdot\left(p_{\mathrm{f}}^{(0)} \frac{\tilde{K}_{\mathrm{f}}^{*}}{\mu} \vec{\nabla}_{x} p_{\mathrm{f}}^{(0)}\right)=0, \\
& \text { - Model } \mathrm{III}_{\mathrm{g}}^{*}(n>2) \\
& \phi_{\mathrm{f}} \frac{\partial p_{\mathrm{f}}^{(0)}}{\partial t}-\vec{\nabla}_{x} \cdot\left(p_{\mathrm{f}}^{(0)} \frac{\tilde{K}_{\mathrm{f}}}{\mu} \vec{\nabla}_{x} p_{\mathrm{f}}^{(0)}\right)=0 .
\end{aligned}
$$

In model $\mathrm{II}_{\mathrm{g}}^{*}$, the $\Omega$-periodic pressure $p_{\mathrm{m}}^{(0)}$ satisfies

$$
\begin{aligned}
& \phi_{\mathrm{m}} \frac{\partial p_{\mathrm{m}}^{(0)}}{\partial t}-\vec{\nabla}_{y} \cdot\left(D_{\mathrm{m}_{\mathrm{g}}} \vec{\nabla}_{y} p_{\mathrm{m}}^{(0)}\right)=0 \text { in } \Omega_{\mathrm{m}}, \\
& p_{\mathrm{m}}^{(0)}=p_{\mathrm{f}}^{(0)} \quad \text { on } \Gamma .
\end{aligned}
$$

In these models the effective permeability is a fracture gas permeability, $K_{\mathrm{f}_{\mathrm{g}}}^{*}$ which does not satisfy Klinkenberg's law and is characterized by a non-linear relation with respect to $K n_{\mathrm{f}}$ (for details, see Appendix C). The above models are therefore strongly non-linear. The domains of validity are defined by means of values of $n$ only, which results from the fact that the local regimes being considered occur only when $q=-1$. The above behaviors do not describe neither a macroscopic Klinkenberg's regime nor a macroscopic Knudsen's regime but characterize a transition regime between both.

\section{Comments and interpretation}

\subsection{Introduction}

In the present section, we consider the three models presented in Section 3 obtained in Darcy's regime by (Royer and Auriault 1994) together with the six new models derived in Klinkenberg's and Knudsen's regimes (see Section 4.5), so as to analyze globally the behaviors and the notable properties of gas flow in dual-porosity media. Because of fracture flow predominance, each of these nine models results from the volume average of fracture flow mass-balance equation and includes or not a term that reflects the influence of matrix flow. This influence is nonexistent in models $\mathrm{III}_{1}$, $\mathrm{III}_{\mathrm{g}}$ and $\mathrm{III}_{\mathrm{g}}^{*}$ (models of type III), while it is minor in models $\mathrm{I}_{1}, \mathrm{I}_{\mathrm{g}}$ and $\mathrm{I}_{\mathrm{g}}^{*}$ (models of type $\mathrm{I}$ ) and it leads to strong memory effects in models $\mathrm{II}_{1}, \mathrm{II}_{\mathrm{g}}$ and $\mathrm{II}_{\mathrm{g}}^{*}$ (models of type II). Furthermore, the effective permeability of each model is the fracture effective 
permeability: $\tilde{K}_{\mathrm{f}_{\mathrm{f}}}$ in models $\mathrm{I}_{1}, \mathrm{II}_{1}$ and $\mathrm{III}_{1}, \tilde{K}_{\mathrm{f}_{\mathrm{g}}}$ in models $\mathrm{I}_{\mathrm{g}}, \mathrm{II}_{\mathrm{g}}$ and $\mathrm{III}_{\mathrm{g}}$ and $\tilde{K}_{\mathrm{f}}$ in models $\mathrm{I}_{\mathrm{g}}^{*}, \mathrm{II}_{\mathrm{g}}^{*}$ and $\mathrm{III}_{\mathrm{g}}^{*}$. The three effective permeabilities characterize three distinct macroscopic regimes. Below, we successively examine the types of macroscopic behaviors (characterized by the types of models) and the types of macroscopic regimes (given by the effective permeability) described by the models. We then deduce a clear interpretation of their domains of validity.

5.2 Types of macroscopic behaviors

The nine models reveal the existence of three types of macroscopic behaviors

- Type I: Reservoir effect

$\phi^{*} \frac{\partial p}{\partial t}-\vec{\nabla}_{x} \cdot\left(p \frac{\tilde{K}_{\mathrm{f}}}{\mu} \vec{\nabla}_{x} p\right)=\mathcal{O}(\varepsilon)$,

- Type II: Dual-porosity behavior

$\phi_{\mathrm{f}} \frac{\partial p_{\mathrm{f}}}{\partial t}+\phi_{\mathrm{m}} \frac{\partial\left\langle p_{\mathrm{m}}\right\rangle}{\partial t}-\vec{\nabla}_{x} \cdot\left(p_{\mathrm{f}} \frac{\tilde{K}_{\mathrm{f}}}{\mu} \vec{\nabla}_{x} p_{\mathrm{f}}\right)=\mathcal{O}(\varepsilon)$,

- Type III: Single-porosity behavior

$$
\phi_{\mathrm{f}} \frac{\partial p_{\mathrm{f}}}{\partial t}-\vec{\nabla}_{x} \cdot\left(p_{\mathrm{f}} \frac{\tilde{K}_{\mathrm{f}}}{\mu} \vec{\nabla}_{x} p_{\mathrm{f}}\right)=\mathcal{O}(\varepsilon)
$$

in which $\phi_{\mathrm{f}}, \tilde{K}_{\mathrm{f}}$ and $p_{\mathrm{f}}$ represent the porosity, the effective permeability and the pressure in the fractures, respectively. In models of type I, $\phi^{*}$ denotes the porosity of the entire fractured porous medium $\left(\phi^{*}=\phi_{\mathrm{f}}+\phi_{\mathrm{m}}\left(1-\phi_{\mathrm{f}}\right)\right)$ and $p$ is the pressure, with $p=p_{\mathrm{f}}=p_{\mathrm{m}}$. Models of type II are two-pressure field models $\left(p_{\mathrm{m}} \neq p_{\mathrm{f}}\right)$. The matrix pressure field satisfies the boundary-value problem that corresponds to the local matrix flow regime being considered

$$
\begin{aligned}
& \phi_{\mathrm{m}} \frac{\partial p_{\mathrm{m}}}{\partial t}-\vec{\nabla}_{y} \cdot\left(p_{\mathrm{m}} C_{\mathrm{m}} \vec{\nabla}_{y} p_{\mathrm{m}}\right)=0 \text { in } \Omega_{\mathrm{m}}, \\
& p_{\mathrm{m}}=p_{\mathrm{f}} \text { on } \Gamma,
\end{aligned}
$$

where $C_{\mathrm{m}}=K_{\mathrm{m}_{\mathrm{g}}} / \mu, K_{\mathrm{m}_{\mathrm{g}}} / \mu$ or $D_{\mathrm{m}_{\mathrm{g}}} / p_{\mathrm{m}}$. When the micropores are connected, the above boundary-value problem is completed with the condition of $\Omega$-periodicity of $p_{\mathrm{m}}$. Both pressure fields are such that $p_{\mathrm{m}}=\mathcal{F}\left(p_{\mathrm{f}}\right)$, where $\mathcal{F}$ is non-linear functional with memory effects. Models of type II cannot be expressed as a two-equation model with a matrix-fracture exchange term. The domains of validity of these three types of macroscopic behaviors are reported on a $K n_{\mathrm{p}}-\alpha$ diagram in Fig. 3, which reveals the existence of a dual-porosity curve that divides the whole $K n_{\mathrm{p}}-\alpha$ space into two parts: the "reservoir effect" zone and the single-porosity zone. The shape of the dualporosity curve reflects that within the local Darcy's regime zone, the type of behavior depends only on the medium geometry, characterized by the value of $n$, while in the local Klinkenberg's regime zone, the type of behavior depends on both $n$ and $q$, which indicates that besides depending on the relationship between both scale ratios, the macroscopic behavior is also conditioned by the Knudsen number in the micropores. The vertical dashed line drawn on the scheme crosses the three behaviors zones. This shows that, depending on the local Knudsen number being considered in the 


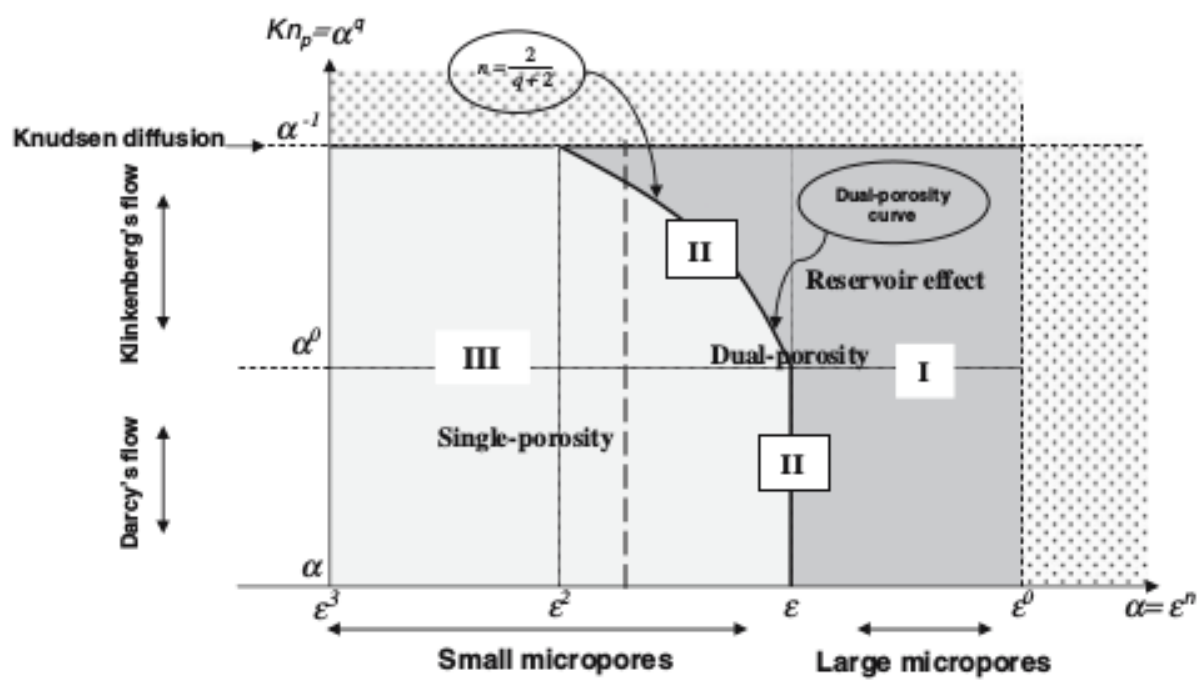

Fig. $3 K n_{\mathrm{p}}-\alpha$ diagram of macroscopic types of behaviors

micropores, any fractured porous medium of geometry characterized by a value of $n$ ranging from 1 to 2 can have the three types of behaviors. In particular, we note that a medium with a single-porosity behavior in local Darcy's regime can have a dual-porosity behavior in local Klinkenberg's regime.

\subsection{Types of macroscopic regimes}

The nine models describe three types of macroscopic regimes, each of which is characterized by a specific effective permeability. We may firstly underline that, as a result of fracture flow predominance, these three effective permeabilities are permeabilities of the fracture network. These three permeabilities are the intrinsic permeability $\tilde{K}_{\mathrm{f}_{\mathrm{l}}}$, the Klinkenberg's gas permeability $\tilde{K}_{\mathrm{f}_{\mathrm{g}}}$ and a non-linear gas permeability $\tilde{K}_{\mathrm{f}_{\mathrm{g}}}^{*}$ (their definitions are presented in Appendix C) and they characterize three types of macroscopic regimes, namely, Darcy's regime $\left(K_{\mathrm{f}_{\mathrm{l}}}\right)$, Klinkenberg's regime $\left(\widetilde{K}_{\mathrm{f}_{\mathrm{g}}}\right)$ and a transition regime between Klinkenberg' and Knudsen's regimes, characterized by $\tilde{K}_{\mathrm{f}_{\mathrm{g}}}^{*}$. Fig. 4 shows their domains of validity and reflects the existence of a gas-permeability curve above which the effective permeability of the fractured porous medium is a gas permeability. It also shows that the intrinsic permeability zone is not limited to the local Darcy's regime zone. As a result, the microporosity may have a notable influence on the effective permeability, and therefore on the type of macroscopic regime. In effect, consider the horizontal dashed line drawn in Fig. 4. We note that it crosses both the intrinsic permeability zone $\left(\tilde{K}_{\mathrm{f}_{\mathrm{f}}}\right)$ and the Klinkenberg's gas permeability zone $\left(\tilde{K}_{\mathrm{f}_{\mathrm{g}}}\right)$. This means that on that line, the effective permeability depends on the microporosity size: the larger the micropore size, the lower the required Knudsen number for getting a macroscopic Klinkenberg's effect.

\subsection{Domains of validity of macroscopic models}

There exist nine combinations between the domains of validity of the types of macroscopic behaviors (Fig. 3) and those of the effective permeabilities (Fig. 4). These nine 


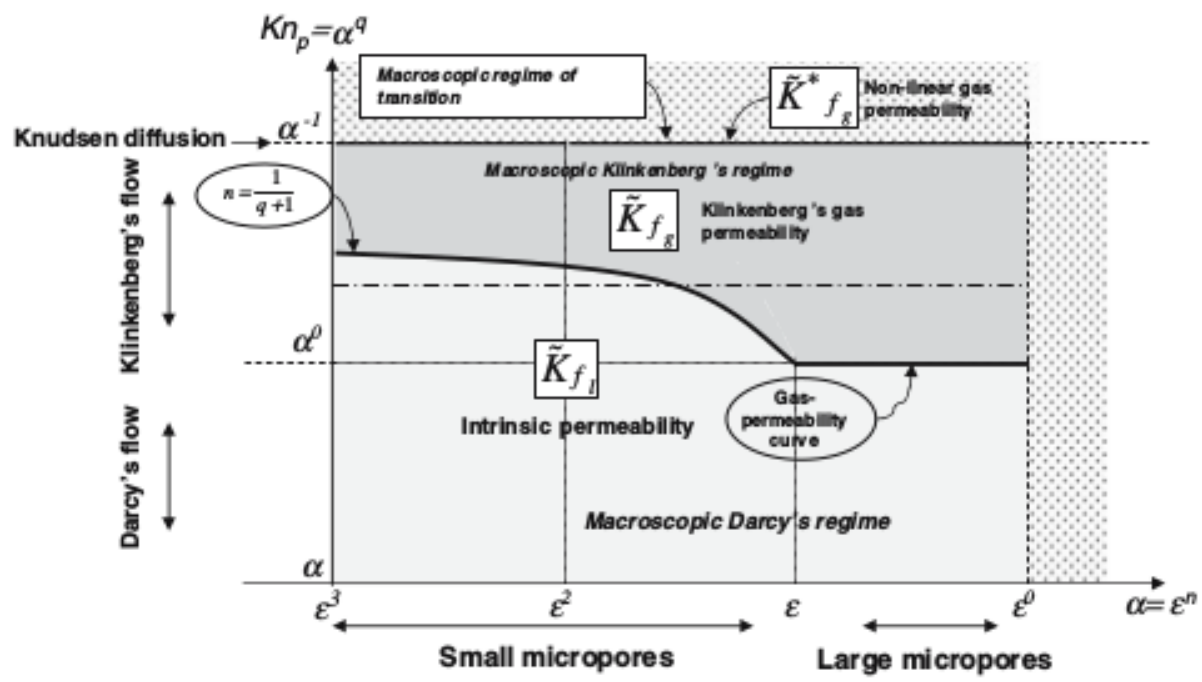

Fig. $4 K n_{\mathrm{p}}-\alpha$ diagram of macroscopic flow regimes

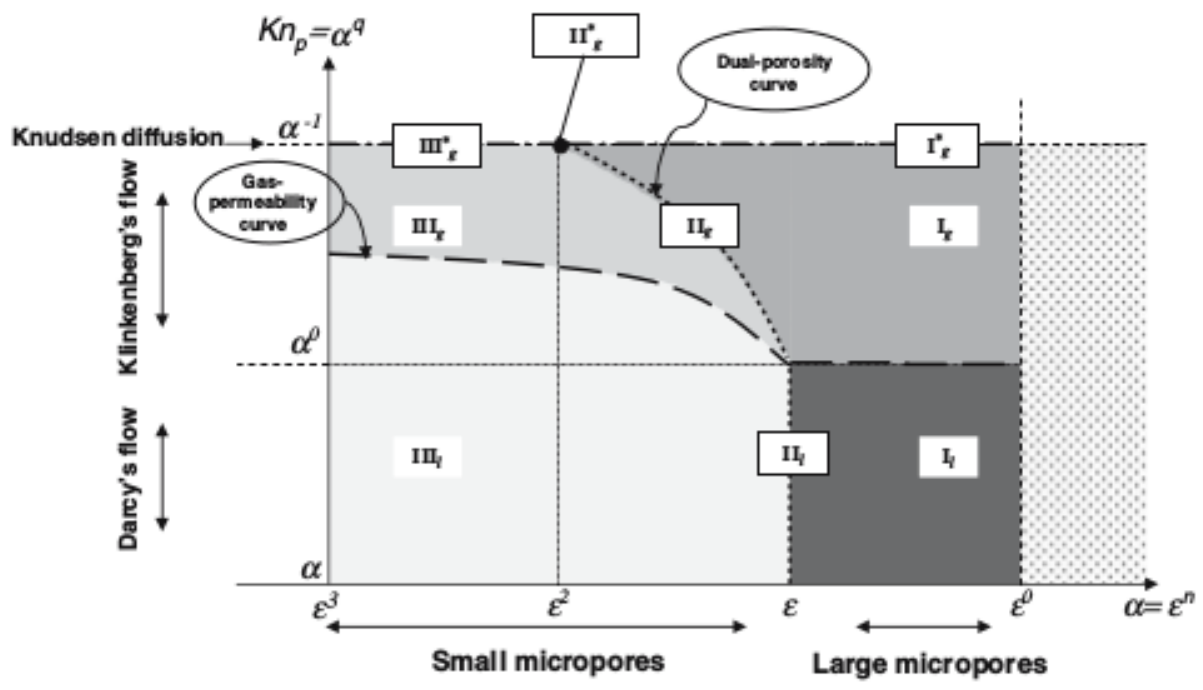

Fig. 5 Domains of validity of macroscopic models

combinations correspond to the domains of validity of the nine macroscopic models and are shown in Fig. 5. Dual-porosity models are located on the dual-porosity curve. Reservoir effect models are located on the right-hand side of the dual-porosity curve and correspond to large micropores, while single-porosity models are located on the left-hand side and correspond to small micropores. Models located above the gas permeability curve include an effective gas permeability. 


\section{Conclusions}

We have applied homogenization theory so as to derive continuum macroscopic models that describe flow of gas at low pressure in dual-porosity media. Considering the case of a fractured porous medium with either Klinkenberg's or Knudsen's diffusion local flow regimes in the porous matrix, we have derived six new macroscopic models that we have compared to the three models obtained in Darcy's regime (Royer and Auriault 1994). Each of these nine models is characterized by its effective permeability and the type of macroscopic behavior it describes. An important conclusion that may be drawn from this study is that not only the domains of validity of models depend on the relative size of pore and fracture scales, but they may even be subordinate to the pore-scale Knudsen number. It follows that for the flow of low pressure gas in dual-porosity media, the macroscopic flow regime, i.e. the effective permeability, may vary with respect to the pore size, and that the type of macroscopic behavior may change with the local flow regime being considered.

\section{Appendix A: Order-of-magnitude of velocity ratio}

The macroscopic sample, of size $L$, is subjected to a macroscopic pressure drop $\delta p_{\mathrm{f}}$, which induces a flow of characteristic velocity $V_{\mathrm{f}}$ and of characteristic time $T_{\mathrm{f}}$ in the fractures, such that

$$
V_{\mathrm{f}}=\mathcal{O}\left(\frac{\delta p_{\mathrm{f}} l^{2}}{\mu_{\mathrm{c}} L}\right), \quad T_{\mathrm{f}}=\mathcal{O}\left(\frac{L}{V_{\mathrm{f}}}\right) .
$$

Because the fracture flow is predominant with regard to the matrix flow, $T_{\mathrm{f}}$ also represents the macroscopic characteristic time of flow. Let now consider the flow in the micropores. It results from a pressure drop, $\delta p_{\mathrm{m}}$, which is applied to the bounds of the matrix domain. The characteristic gas velocity in the matrix may thus be expressed as

$$
V_{\mathrm{m}}=\mathcal{O}\left(K n_{\mathrm{p}} \frac{\delta p_{\mathrm{m}} l_{\mathrm{p}}^{2}}{\mu_{\mathrm{c}} l}\right) .
$$

Let denote by $p_{\mathrm{f}}$ the pressure at a given point within the fracture's domain and which is such that $p_{\mathrm{f}}=\mathcal{O}\left(\delta p_{\mathrm{f}}\right)$. Due to the condition of continuity of pressures, we may consider that the pressure $p_{\mathrm{f}}$ is applied on the matrix/fracture interface. Thus, the pressure drop $\delta p_{\mathrm{m}}$ is actually due to the space variations of $p_{\mathrm{f}}$ over the matrix domain of size $l$. Hence, from the mass-balance equation we shall define the characteristic time of transit in the matrix, $T_{\mathrm{m}}^{\mathrm{t}}$, by

$$
\frac{p_{\mathrm{f}}}{T_{\mathrm{m}}^{\mathrm{t}}}=\mathcal{O}\left(\frac{p_{\mathrm{f}} V_{\mathrm{m}}}{l}\right) \quad \text { or } \quad T_{\mathrm{m}}^{\mathrm{t}}=\mathcal{O}\left(\frac{l}{V_{\mathrm{m}}}\right) .
$$

Since the preponderant flow is the fracture flow, $T_{\mathrm{m}}^{\mathrm{t}}$ is necessarily greater or equal than its counterpart in the fractures, namely $T_{\mathrm{f}}$

$$
T_{\mathrm{m}}^{\mathrm{t}} \geq T_{\mathrm{f}} .
$$

Now, the pressure $p_{\mathrm{f}}$ exerted on the matrix/fracture boundary is also subjected to time variations. Let now denote by $p_{\mathrm{m}}$ the pressure at a given point within the matrix 
domain, which is such that $p_{\mathrm{m}}=\mathcal{O}\left(\delta p_{\mathrm{m}}\right)$. The time variations of $p_{\mathrm{f}}$ induce time variations of the local pressure $p_{\mathrm{m}}$. The issue is to determine whether these time variations of the matrix pressure may occur within the whole matrix domain or not. Let consider that these time variations occur in a boundary layer of thickness $l^{*}$, which is such that

$$
l^{*} \leq l,
$$

and let denote by $T_{\mathrm{m}}^{\mathrm{P}}$ the char acteristic time of pressure variation in the matrix. From the mass-balance equation in the porous matrix we therefore get

$$
\frac{p_{\mathrm{m}}}{T_{\mathrm{m}}^{\mathrm{P}}}=\mathcal{O}\left(\frac{p_{\mathrm{m}} V_{\mathrm{m}}}{l^{*}}\right) \quad \text { or } \quad T_{\mathrm{m}}^{\mathrm{P}}=\mathcal{O}\left(\frac{l^{*}}{V_{\mathrm{m}}}\right) .
$$

In regard to the definitions of $T_{\mathrm{m}}^{\mathrm{P}}$ and of $T_{\mathrm{m}}^{\mathrm{t}}$, (A.6) and (A.3), we have from (A.5)

$$
T_{\mathrm{m}}^{\mathrm{t}} \geq T_{\mathrm{m}}^{\mathrm{P}},
$$

which means that the pressure time variations occur instantaneously within the boundary layer. We have thus obtained two estimates for the time variations of pressure within the matrix domain, $p_{\mathrm{f}} / T_{\mathrm{m}}^{\mathrm{t}}$ and $p_{\mathrm{m}} / T_{\mathrm{m}}^{\mathrm{P}}$, whence it follows that

$$
\frac{p_{\mathrm{m}}}{T_{\mathrm{m}}^{\mathrm{P}}}=\mathcal{O}\left(\frac{p_{\mathrm{f}}}{T_{\mathrm{m}}^{\mathrm{t}}}\right) .
$$

Let now examine the roles played by the distinct characteristic times. An important feature of dual-porosity media is that the matrix flow will be visible at the macroscopic scale provided that the characteristic time of transit in the matrix is equal to the macroscopic characteristic time of transit, $T_{\mathrm{m}}^{\mathrm{t}}=T_{\mathrm{f}}$. We deduce that if $T_{\mathrm{m}}^{\mathrm{t}} \neq T_{\mathrm{m}}^{\mathrm{P}}$, then, as the matrix flow is not visible macroscopically, the pressure time variations occur within the whole matrix. This happens when $l^{*}=l$, i.e. when $T_{\mathrm{m}}^{\mathrm{t}}=T_{\mathrm{m}}^{\mathrm{P}}$. We thus deduce that $T_{\mathrm{m}}^{\mathrm{t}}$ is either equal to $T_{\mathrm{f}}$ or to $T_{\mathrm{m}}^{\mathrm{P}}$ :

$$
\left\{\begin{array}{l}
T_{\mathrm{m}}^{\mathrm{t}}=T_{\mathrm{f}}, \\
\text { or } \\
T_{\mathrm{m}}^{\mathrm{t}}=T_{\mathrm{m}}^{\mathrm{P}}
\end{array}\right.
$$

From inequalities (A.7) and (A.4) together with (A.9), it turns out that three situations may occur

$$
\begin{cases}\text { I. } & T_{\mathrm{m}}^{\mathrm{P}}<T_{\mathrm{f}}=T_{\mathrm{m}}^{\mathrm{t}} \\ \text { II. } & T_{\mathrm{m}}^{\mathrm{P}}=T_{\mathrm{f}}=T_{\mathrm{m}}^{\mathrm{t}} \\ \text { III. } & T_{\mathrm{f}}<T_{\mathrm{m}}^{\mathrm{P}}=T_{\mathrm{m}}^{\mathrm{t}}\end{cases}
$$

As they correspond to three combinations between the characteristic times, we thus deduce that the three above defined cases lead to three distinct types of macroscopic behaviors. Let now determine the order of magnitude of $T_{\mathrm{m}}^{\mathrm{P}} / T_{\mathrm{f}}$. From the definitions of $T_{\mathrm{m}}^{\mathrm{P}}$ and $T_{\mathrm{f}}$ and by noticing from (A.8) that $\delta p_{\mathrm{f}} / \delta p_{\mathrm{m}}=\mathcal{O}\left(l / l^{*}\right)$, we get

$$
\frac{T_{\mathrm{m}}^{\mathrm{P}}}{T_{\mathrm{f}}}=\mathcal{O}\left(K n_{\mathrm{p}}^{-1} \alpha^{-2} \varepsilon^{2}\right)=\mathcal{O}\left(\varepsilon^{-n(q+2)+2}\right) .
$$

Considering (A.10) and (A.11), we are now able to determine the possible orders of magnitude for $\gamma=\mathcal{O}\left(V_{\mathrm{m}} / V_{\mathrm{f}}\right)$. When $T_{\mathrm{m}}^{\mathrm{P}} \leq T_{\mathrm{f}}(n \leq 2 / q+2)$, the result is obtained by considering the definitions of $T_{\mathrm{f}}$ and of $T_{\mathrm{m}}^{\mathrm{t}}$, while taking into account the equality 
$T_{\mathrm{f}}=T_{\mathrm{m}}^{\mathrm{t}}$. We get $\gamma=\mathcal{O}(\varepsilon)$. When $T_{\mathrm{m}}^{\mathrm{P}}>T_{\mathrm{f}}(n>2 / q+2)$, the order of magnitude of $\gamma$ is deduced from the orders of $V_{\mathrm{m}}$ and $V_{\mathrm{f}}$, (A.2) and (A.1), and from estimation (A.8), while considering $T_{\mathrm{m}}^{\mathrm{P}}=T_{\mathrm{m}}^{\mathrm{t}}$. We obtain $\gamma=\mathcal{O}\left(K n_{\mathrm{p}} \alpha^{2} \varepsilon^{-1}\right)$. Finally, the order-of-magnitude of the velocity ratio is such that

$$
\gamma=\frac{V_{\mathrm{m}}}{V_{\mathrm{f}}}=\left\{\begin{array}{lll}
\mathcal{O}(\varepsilon) & \text { if } & n \leq \frac{2}{q+2}, \\
\mathcal{O}(\varepsilon) & \text { if } & n=\frac{2}{q+2} \\
\mathcal{O}\left(K n_{\mathrm{p}} \alpha^{2} \varepsilon^{-1}\right) & \text { if } & n \geq \frac{2}{q+2}
\end{array}\right.
$$

\section{Appendix B. Derivation of Model $\mathbf{I}_{\mathrm{g}}$}

We consider the case $n=2 / q+2$ in the dimensionless local description (4.34)-(4.39) and we look for variables $p_{\mathrm{f}}, \vec{v}_{\mathrm{f}}, p_{\mathrm{m}}, \vec{V}_{\mathrm{m}}, K_{\mathrm{m}_{\mathrm{g}}}$ and $\vec{t}_{1}$ in the form (4.40). The method consists then in introducing these asymptotic expansions in the dimensionless description and in identifying at the successive orders of $\varepsilon$. Let now determine and solve the appropriate approximate boundary-value problems. We begin by considering Equation (4.36) at the order $\mathcal{O}\left(\varepsilon^{-1}\right): \vec{\nabla}_{y} p_{\mathrm{f}}^{(0)}=\overrightarrow{0}$. It leads to $p_{\mathrm{f}}^{(0)}=p_{\mathrm{f}}^{(0)}(\vec{x}, t)$. Next we consider Equations (4.34), (4.35) and (4.39) at the first order

$$
\begin{aligned}
& \phi_{\mathrm{m}} \frac{\partial p_{\mathrm{m}}^{(0)}}{\partial t}-\vec{\nabla}_{y} \cdot\left(p_{\mathrm{m}}^{(0)} \frac{K_{\mathrm{m}}^{(0)}}{\mu} \vec{\nabla}_{y} p_{\mathrm{m}}^{(0)}\right)=0 \text { in } \Omega_{\mathrm{m}}, \\
& p_{\mathrm{m}}^{(0)}=p_{\mathrm{f}}^{(0)} \text { on } \Gamma, \\
& p_{\mathrm{m}}^{(0)}: \vec{y} \text {-periodic }
\end{aligned}
$$

from which we deduce $p_{\mathrm{m}}^{(0)}=p_{\mathrm{m}}^{(0)}(\vec{y}, \vec{x}, t)$. Then, considering Equation (4.36) at the order $\mathcal{O}\left(\varepsilon^{0}\right)$, Equation (4.37) at the order $\mathcal{O}\left(\varepsilon^{-1}\right)$ and boundary-condition (4.39) at the order $\mathcal{O}\left(\varepsilon^{0}\right)$, we obtain the following boundary-value problem for the first-order velocity field in the fracture's domain

$$
\begin{aligned}
& \mu \Delta_{y} \vec{v}_{\mathrm{f}}^{(0)}-\vec{\nabla}_{y} p_{\mathrm{f}}^{(1)}-\vec{\nabla}_{x} p_{\mathrm{f}}^{(0)}=\overrightarrow{0} \quad \text { in } \Omega_{\mathrm{f}}, \\
& \vec{\nabla}_{y} \cdot \vec{v}_{\mathrm{f}}^{(0)}=0 \quad \text { in } \Omega_{\mathrm{f}}, \\
& \vec{v}_{\mathrm{f}}^{(0)}=-K n_{\mathrm{f}}\left(\vec{t}_{1}^{(0)} \cdot \vec{\nabla}_{y} \vec{v}_{\mathrm{f}}^{(0)} \cdot n\right) \vec{t}_{1}^{(0)} \quad \text { on } \Gamma
\end{aligned}
$$

in which $\vec{v}_{\mathrm{f}}^{(0)}, p_{\mathrm{f}}^{(1)}$ and $\vec{t}_{1}^{(0)}$ are $\vec{y}$-periodic. The solution is expressed as $\vec{v}_{\mathrm{f}}^{(0)}=$ $-\tilde{K}_{\mathrm{f}_{\mathrm{g}}} / \mu \vec{\nabla}_{x} p_{\mathrm{f}}^{(0)}$, and its average over the period yields

$$
\left\langle\vec{v}_{\mathrm{f}}^{(0)}\right\rangle_{\Omega}=-\frac{\tilde{K}_{\mathrm{f}_{\mathrm{g}}}}{\mu} \vec{\nabla}_{x} p_{\mathrm{f}}^{(0)}, \quad \tilde{K}_{\mathrm{f}_{\mathrm{g}}}=\left\langle\tilde{K}_{\mathrm{f}_{\mathrm{g}}}\right\rangle_{\Omega}
$$

As in (Skjetne and Auriault 1999), $\vec{v}_{\mathrm{f}}^{(0)}$ and $p_{\mathrm{f}}^{(1)}$ are then looked in the form of asymptotic expansions of $K n_{\mathrm{f}}$ and these expansions are substituted into boundaryvalue problem (B.14). By considering the first two leading orders, we obtain that $\tilde{K}_{\mathrm{f}_{\mathrm{g}}}=\tilde{K}_{\mathrm{f}_{\mathrm{f}}}+K n \tilde{k}_{\mathrm{f}_{\mathrm{g}}}^{1}\left(\tilde{K}_{\mathrm{f}_{\mathrm{l}}}\right.$ and $\tilde{k}_{\mathrm{f}_{\mathrm{g}}}^{1}$ are defined in Appendix C). We thus deduce that $\tilde{K}_{\mathrm{f}_{\mathrm{g}}}=\tilde{K}_{\mathrm{f}_{\mathrm{l}}}\left(\tilde{I}+K n_{\mathrm{f}} \tilde{C}\right)$, where $\tilde{C}=\left(\tilde{K}_{\mathrm{f}_{l}}\right)^{-1} \tilde{K}_{\mathrm{f}_{\mathrm{g}}}^{1}$ and where $K_{\mathrm{f}_{\mathrm{g}_{i j}}}^{1}=\left\langle k_{\mathrm{f}_{8 j}}^{1}\right\rangle_{\Omega}$. Let finally 
consider the first-order mass-balance equations together with the projection on surface normal of boundary-condition (4.39) at the second order

$$
\begin{aligned}
& \frac{\partial p_{\mathrm{f}}^{(0)}}{\partial t}+\vec{\nabla}_{y} \cdot\left(p_{\mathrm{f}}^{(1)} \vec{v}_{\mathrm{f}}^{(0)}+p_{\mathrm{f}}^{(0)} \vec{v}_{\mathrm{f}}^{(1)}\right)+\vec{\nabla}_{x} \cdot\left(p_{\mathrm{f}}^{(0)} \vec{v}_{\mathrm{f}}^{(0)}\right)=0 \text { in } \Omega_{\mathrm{f}}, \\
& \phi_{\mathrm{m}} \frac{\partial p_{\mathrm{m}}^{(0)}}{\partial t}+\vec{\nabla}_{y} \cdot\left(p_{\mathrm{m}}^{(0)} \vec{v}_{\mathrm{m}}^{(0)}\right)=0 \quad \text { in } \Omega_{\mathrm{m}}, \\
& \vec{v}_{\mathrm{f}}^{(1)} \cdot \vec{n}=\vec{v}_{\mathrm{m}}^{(0)} \cdot \vec{n} \quad \text { on } \Gamma,
\end{aligned}
$$

where $\vec{v}_{\mathrm{f}}^{(1)}, \vec{v}_{\mathrm{m}}^{(0)}$ are $\vec{y}$-periodic. Integrating the above boundary-value problem over the period while using the divergence theorem yields the macroscopic model

$$
\phi_{\mathrm{f}} \frac{\partial p_{\mathrm{f}}^{(0)}}{\partial t}+\phi_{\mathrm{m}} \frac{\partial\left\langle p_{\mathrm{m}}^{(0)}\right\rangle}{\partial t}-\vec{\nabla}_{x} \cdot\left(p_{\mathrm{f}}^{(0)} \frac{\tilde{K}_{\mathrm{f}_{\mathrm{g}}}}{\mu} \vec{\nabla}_{x} p_{\mathrm{f}}^{(0)}\right)=0 .
$$

\section{Appendix C: Definitions of effective permeabilities}

C.1 Intrinsic permeability $\tilde{K}_{\mathrm{f}_{\mathrm{l}}}$

Tensor $\tilde{K}_{\mathrm{f}_{1}}$ in models $\mathrm{I}_{1}, \mathrm{II}_{1}$ and $\mathrm{III}_{1}$ is the effective intrinsic permeability of the fracture network and is defined by

$$
K_{\mathrm{f}_{\mathrm{ij}}}=\left\langle k_{\mathrm{f}_{\mathrm{lij}}}\right\rangle_{\Omega}=\frac{1}{|\Omega|} \int_{\Omega_{\mathrm{f}}} k_{\mathrm{f}_{\mathrm{i} i j}} d \Omega,
$$

where $K_{\mathrm{f}_{i j}}$ satisfies

$$
\begin{aligned}
& \frac{\partial^{2} k_{\mathrm{f}_{l_{i j}}}}{\partial y_{i}^{2}}-\frac{\partial \kappa_{\mathrm{f}_{\mathrm{l}_{j j}}}}{\partial y_{i}}+\delta_{i j}=0 \quad \text { in } \Omega_{\mathrm{f}}, \\
& \frac{\partial k_{\mathrm{f}_{\mathrm{i}_{i j}}}}{\partial y_{i}}=0 \quad \text { in } \Omega_{\mathrm{f}}, \\
& k_{\mathrm{f}_{\mathrm{f}_{i j}}}=0 \quad \text { on } \Gamma, \\
& k_{\mathrm{f}_{\mathrm{f}_{i j}}}, \kappa_{\mathrm{f}_{\mathrm{f}_{i j}}}: \Omega \text {-periodic. }
\end{aligned}
$$

It may be shown (Ene and Sanchez-Palencia 1975; Sanchez-Palencia 1980) that $\tilde{K}_{\mathrm{f}_{\mathrm{l}}}$ is a symmetrical and positive second-rank tensor.

C.2 Klinkenberg's gas permeability $\tilde{K}_{\mathrm{f}_{\mathrm{g}}}$

Tensor $\tilde{K}_{\mathrm{f}_{\mathrm{g}}}$ in models $\mathrm{I}_{\mathrm{g}}, \mathrm{II}_{\mathrm{g}}$ and $\mathrm{II}_{\mathrm{g}}$ is such that $K_{\mathrm{f}_{b i j}}=\left\langle K_{\mathrm{f}_{\overline{g i j}}}\right\rangle_{\Omega}$, where $\tilde{K}_{\mathrm{f}_{\mathrm{g}}}=$ $\tilde{K}_{\mathrm{f}_{\mathrm{l}}}+K n \tilde{k}_{\mathrm{f}_{\mathrm{g}}}^{1}$. Tensor $K_{\mathrm{f}_{\mathrm{i} i j}}$ is defined above and $k_{\mathrm{f}_{b i j}}^{1}$ satisfies the boundary-value problem (C.18)-(C.21), but in which Equation (C.20) has been replaced by

$$
k_{\mathrm{f}_{b i j}}^{1}=-\left(t_{1_{i}}^{(0)} \frac{\partial k_{\mathrm{f}_{\delta_{i j}}}^{1}}{\partial y_{i}} n_{i}\right) t_{1_{j}}^{(0)} \quad \text { on } \Gamma .
$$


Consequently, tensor $\tilde{K}_{\mathrm{fg}}$ can be written as

$$
\tilde{K}_{\mathrm{f}_{\mathrm{g}}}=\tilde{K}_{\mathrm{f}_{\mathrm{l}}}\left(\tilde{I}+K n_{\mathrm{f}} \tilde{C}\right),
$$

where $\tilde{C}$ is a positive tensor (Skjetne and Auriault 1999). It is defined by $\tilde{C}=$ $\left(\tilde{K}_{\mathrm{f}_{l}}\right)^{-1} \tilde{K}_{\mathrm{f}_{\mathrm{g}}}^{1}$, where $K_{\mathrm{f}_{\delta_{i j}}}^{1}=\left\langle k_{\mathrm{f}_{\mathrm{g}_{i j}}}^{1}\right\rangle_{\Omega}$. Equation (C.23) is the tensorial form of Klinkenberg's law.

\section{C.3 Non-Linear gas permeability $\tilde{K}_{\mathrm{f}_{\mathrm{g}}}^{*}$}

Tensor $\tilde{K}_{\mathrm{f}_{\mathrm{g}}}^{*}$ in models $\mathrm{I}_{\mathrm{g}}^{*}$, $\mathrm{II}_{\mathrm{g}}^{*}$ and $\mathrm{III}_{\mathrm{g}}^{*}$ is such that $K_{\mathrm{f}_{\mathrm{g} j}}^{*}=\left\langle k_{\mathrm{f}_{\mathrm{gij}}}^{*}\right\rangle_{\Omega}$, where $k_{\mathrm{g}_{j j}}^{*}$ is the solution to the boundary-value problem (C.18)-(C.21), but in which Equation (C.20) has been replaced by

$$
k_{\mathrm{f}_{\delta i j}}^{*}=-K n_{\mathrm{f}}\left(t_{1_{i}}^{(0)} \frac{\partial k_{\mathrm{f}_{z i j}}^{*}}{\partial y_{i}} n_{i}\right) t_{1_{j}}^{(0)} \quad \text { on } \Gamma .
$$

Therefore, $\tilde{K}_{\mathrm{f}_{\mathrm{g}}}^{*}$ depends on $K n_{\mathrm{f}}$, but the relation between both is non-linear.

\section{References}

Adzumi, H.: Studies on the flow of gaseous mixtures through capillaries: I. The viscosity of binary gaseous mixtures. Bull. Chem. Soc. Japan 12, 199-226 (1937a)

Adzumi, H.: Studies on the flow of gaseous mixtures through capillaries: II. The molecular flow of gaseous mixtures, Bull. Chem. Soc. Japan 12, 285-291 (1937b)

Auriault, J.-L.: Heterogeneous medium. Is an equivalent macroscopic description possible? Int. J. Engng. Sci. 29(7), 785-795 (1991)

Auriault, J.-L.: Transport in porous media: upscaling by multiple asymptotic expansions. In: Dormieux, L., Ulm, F-J. (eds.) CISM Lecture 480 Applied Micromechanics of Porous Materials, pp. 3-56. Springer (2006)

Auriault, J-L., Boutin, C: Deformable porous media with double porosity. Quasi-statics I: Coupling effects, Transport Porous Media 7, 63-82 (1992)

Auriault, J-L., Boutin, C.: Deformable porous media with double por osity. Quasi-statics II: Memory effects. Transport in Porous Media 10, 153-169 (1993)

Barenblatt, G., Zheltov, I., Kochina, I.: Basic concepts in theory of seepage of homogeneous liquids in the fractured rock. J. Appl. Math. Mech. 24, 1286-1303 (1960)

Bensoussan, A., Lions, J-L., Papanicolaou, G.: Asymptotic Analysis for Periodic Structures, NorthHolland Publishing Company, Amsterdam (1978)

Cercignani, C.: The Boltzmann Equation and its Applications. Springer-Verlag, Berlin (1988)

Char rier, P., Dubroca, B.: Asymptotic transport models for heat and mass transfer in reactive porous media. C.R.A.S. Ser. I 336, 537-542 (2003)

Derjaguin, B.: Measurement of specific surface of porous and disperse bodies by their resistance to the flow of rarefied gas, C.R Acad. Sci. URSS 53, 623-626 (1946)

Ene, H.I., Sanchez-palencia, E.: Equations et phénomènes de surface pour l'écoulement dans un modele de milieu poreux. Journal de Mécanique 14(1), 73-108 (1975)

Kast, W., Hohenthanner, C.-R.: Mass transfer within the gas-phase of porous media. Int. J. Mass Transfer 43, 807-823 (2000)

Klinkenberg, L.-J: The permeability of porous media to liquids and gas Drilling and Production Practice, American Petroleum Inst., 200-213 (1941)

Knudsen, M.: Die gesetze der molecularströmung and der inneren reibungsstrŏmung edr gase durch röhren. Annalen der Physik 34, 75-130 (1909)

Moyne, C.: Two-equation model for a diffusive process in porous media using the volume averaging method with unsteady-state closure. Adv. Water Res. 20(2-3), 63-76 (1997) 
Royer, P., Auriault, J.-L.: Transient quasi-static gas flow through a rigid porous medium with double porosity. Transport in Porous Media 17(1), 33-57 (1994)

Royer, P., Auriault, J.-L., Lewandowska J., Serres C.: Continuum modelling of contamin ant transport in fractured porous media. Transport in Porous Media 49, 333-359 (2002)

Sanchez-Palencia, E.: Non-Homogeneous Media and Vibration Theory, Lecture Notes in Physics 127. Springer-Verlag, Berlin (1980)

Skjetne, E., Auriault, J-L.: Homogenization of wall-slip flow through porous media. Transport Porous Media 36(1), 293-306 (1999) 